\title{
Numerical Solutions of Stochastic Differential Equations with Piecewise Continuous Arguments under Khasminskii-Type Conditions
}

\author{
Minghui Song ${ }^{1}$ and Ling Zhang ${ }^{1,2}$ \\ ${ }^{1}$ Department of Mathematics, Harbin Institute of Technology, Harbin 150001, China \\ 2 Institute of Mathematical Sciences, Daqing Normal University, Daqing 163712, China \\ Correspondence should be addressed to Minghui Song, songmh@lsec.cc.ac.cn
}

Received 9 April 2012; Accepted 13 June 2012

Academic Editor: F. Marcellán

Copyright (C) 2012 M. Song and L. Zhang. This is an open access article distributed under the Creative Commons Attribution License, which permits unrestricted use, distribution, and reproduction in any medium, provided the original work is properly cited.

\begin{abstract}
The main purpose of this paper is to investigate the convergence of the Euler method to stochastic differential equations with piecewise continuous arguments (SEPCAs). The classical Khasminskiitype theorem gives a powerful tool to examine the global existence of solutions for stochastic differential equations (SDEs) without the linear growth condition by the use of the Lyapunov functions. However, there is no such result for SEPCAs. Firstly, this paper shows SEPCAs which have nonexplosion global solutions under local Lipschitz condition without the linear growth condition. Then the convergence in probability of numerical solutions to SEPCAs under the same conditions is established. Finally, an example is provided to illustrate our theory.
\end{abstract}

\section{Introduction}

Stochastic modeling has come to play an important role in many branches of science and industry. Such models have been used with great success in a variety of application areas, including biology, epidemiology, mechanics, economics, and finance. Most stochastic differential equations are nonlinear and cannot be solved explicitly, but it is very important to research the existence and uniqueness of solution of stochastic differential equations. Many authors have studied the problem of SDEs. The classical existence-and-uniqueness theorem requires the coefficients $f(x(t))$ and $g(x(t))$ to satisfy the local Lipschitz condition and the linear growth condition (see [1]). However, there are many SDEs that do not satisfy the linear growth condition, so more general conditions have been introduced to replace theirs. Khasminskii [2] has studied Khasminskii's test for SDEs which are the most powerful conditions. Similarly, the classical existence-and-uniqueness theorem for stochastic 
differential delay equations (SDDEs) requires the coefficients $f(x(t), x(t-\tau))$ and $g(x(t), x(t-$ $\tau)$ ) to satisfy the local Lipschitz condition and the linear growth condition (see [3-6]). Mao [7] has proved Khasminskii-type theorem, and this is a natural generalization of the classical Khasminskii test.

In recent years, differential equations with piecewise continuous arguments (EPCAs) had attracted much attention, and many useful conclusions were obtained. These systems have applications in certain biomedical models, control systems with feedback delay in the work of L. Cooke and J. Wiener [8]. The general theory and basic results for EPCAs have by now been thoroughly investigated in the book of Wiener [9]. A typical EPCA contains arguments that are constant on certain intervals. The solutions are determined by a finite set of initial data, rather than by an initial function, as in the case of general functional differential equation. A solution is defined as a continuous, sectionally smooth function that satisfies the equation within these intervals. Continuity of a solution at a point joining any two consecutive intervals leads to recursion relations for the solution at such points. Hence, EPCAs represent a hybrid of continuous and discrete dynamical systems and combine the properties of both differential and difference equations.

However, up to now, there are few people who have considered the influence of noise to EPCAs. Actually, the environment, and accidental events may greatly influence the systems. Thus, analyzing SEPCAs is an interesting topic both in theory and applications. In this paper, we give the Khasminskii-type theorems for SEPCAs, which shows that SEPCAs have nonexplosion global solutions under local Lipschitz condition without the linear growth condition.

On the other hand, there is in general no explicit solution to an SEPCA, whence numerical solutions are required in practice. Numerical solutions to SDEs have been discussed under the local Lipschitz condition and the linear growth condition by many authors (see [5]). Mao [10] gives the convergence in probability of numerical solutions to SDDEs under Khasminskii-Type conditions. Dai and Liu [11] give the mean-square stability of the numerical solutions of linear stochastic differential equations with piecewise continuous arguments. However, SEPCAs do not have the convergence results. The other main aim of this paper is to establish convergence of numerical solution for SEPCAs under the differential conditions.

The paper is organized as follows. In Section 2, we introduce necessary notations and Euler method. In Section 3, we obtain the existence and uniqueness of solution to stochastic differential equations with piecewise continuous arguments under Khasminskiitype conditions. Then the convergence in probability of numerical solutions to stochastic differential equations with piecewise continuous arguments under the same conditions is established. Finally, an example is provided to illustrate our theory.

\section{Preliminary Notation and Euler Method}

In this paper, unless otherwise specified, let $|x|$ be the Euclidean norm in $x \in R^{n}$. If $A$ is a vector or matrix, its transpose is defined by $A^{T}$. If $A$ is a matrix, its trace norm is defined by $|A|=\sqrt{\operatorname{trace}\left(A^{T} A\right)}$. For simplicity, we also have to denote by $a \wedge b=\min \{a, b\}, a \vee b=$ $\max \{a, b\}$.

Let $(\Omega, \mathcal{F}, P)$ be a complete probability space with a filtration $\left\{\mathcal{F}_{t}\right\}_{t \geq 0}$, satisfying the usual conditions. $\mathcal{L}^{1}\left([0, \infty), R^{n}\right)$ and $\mathcal{L}^{2}\left([0, \infty), R^{n}\right)$ denote the family of all real-valued $\mathcal{F}_{t^{-}}$ adapted process $f(t)_{t \geq 0}$, such that for every $T>0, \int_{0}^{T}|f(t)| d t<\infty$ almost surely and 
$\int_{0}^{T}|f(t)|^{2} d t<\infty$ almost surely, respectively. For any $a, b \in R$ with $a<b$, denote $C\left([a, b] ; R^{n}\right)$ as the family of continuous functions $\phi$ from $[a, b]$ to $R^{n}$ with the norm $\|\phi\|=\sup _{a \leq \theta \leq b}|\phi(\theta)|$. Denote $C_{q_{t}}^{b}\left([a, b] ; R^{n}\right)$ as the family of all bounded $\mp_{t}$-measurable $C\left([a, b] ; R^{n}\right)$-valued random variables. Let $B(t)=\left(B_{1}(t), \ldots, B_{d}(t)\right)^{T}$ be a $d$-dimensional Brownian motion defined on the probability space. Let $C^{2}\left(R^{n} ; R_{+}\right)$denote the family of all continuous nonnegative functions $V(x)$ defined on $R^{n}$ such that they are continuously twice differentiable in $x$. Given $V \in C^{2}\left(R^{n} ; R_{+}\right)$, we define the operator $L V: R^{n} \times R^{n} \rightarrow R$ by

$$
L V(x, y)=V_{x}(x) f(x, y)+\frac{1}{2} \operatorname{trace}\left[g^{T}(x, y) V_{x x}(x) g(x, y)\right]
$$

where

$$
V_{x}(x)=\left(\frac{\partial V(x)}{\partial x_{1}}, \ldots, \frac{\partial V(x)}{\partial x_{n}}\right), \quad V_{x x}(x)=\left(\frac{\partial^{2} V(x)}{\partial x_{i} \partial x_{j}}\right)_{n \times n}
$$

Let us emphasize that $L V$ is defined on $R^{n} \times R^{n}$, while $V$ is only defined on $R^{n}$.

Throughout this paper, we consider stochastic differential equations with piecewise continuous arguments

$$
d x(t)=f(x(t), x([t])) d t+g(x(t), x([t])) d B(t) \quad \forall t \geq 0,
$$

with initial data $x(0)=c_{0}$, where $f: R^{n} \times R^{n} \rightarrow R^{n}, g: R^{n} \times R^{n} \rightarrow R^{n \times d}, c_{0}$ is a vector, and $[\cdot]$ denotes the greatest-integer function. By the definition of stochastic differential, this equation is equivalent to the following stochastic integral equation:

$$
x(t)=x(0)+\int_{0}^{t} f(x(s), x([s])) d s+\int_{0}^{t} g(x(s), x([s])) d B(s) \quad \forall t \geq 0 .
$$

Moreover, we also require the coefficients $f(x(t), x([t]))$ and $g(x(t), x([t]))$ to be sufficiently smooth.

To be precise, let us state the following conditions.

(H1) (The local Lipschitz condition) For every integer $i \geq 1$, there exists a positive constant $L_{i}$ such that

$$
|f(x, y)-f(\bar{x}, \bar{y})|^{2} \vee|g(x, y)-g(\bar{x}, \bar{y})|^{2} \leq L_{i}\left(|x-\bar{x}|^{2}+|y-\bar{y}|^{2}\right),
$$

for those $x, \bar{x}, y, \bar{y} \in R^{n}$ with $|x| \vee|\bar{x}| \vee|y| \vee|\bar{y}| \leq i$.

(H2) (Linear growth condition) There exists a positive constant $K$ such that

$$
|f(x, y)|^{2} \vee|g(x, y)|^{2} \leq K\left(1+|x|^{2}+|y|^{2}\right)
$$

for all $(x, y) \in R^{n} \times R^{n}$. 
(H3) There are a function $V \in C^{2}\left(R^{n} ; R_{+}\right)$and a positive constant $\alpha$ such that

$$
\begin{gathered}
\liminf _{|x| \rightarrow \infty} V(x)=\infty \\
L V(x, y) \leq \alpha(1+V(x)+V(y)),
\end{gathered}
$$

for all $(x, y) \in R^{n} \times R^{n}$.

Let us first give the definition of the solution.

Definition 2.1 (see [11]). An $R^{n}$-valued stochastic process $\{x(t)\}$ is called a solution of $(2.3)$ on $[0, \infty)$ if it has the following properties:

(1) $\{x(t)\}$ is continuous on $[0, \infty)$ and $\mathcal{F}_{t}$-adapted,

(2) $\{f(x(t), x([t]))\} \in \mathcal{L}^{1}\left([0, \infty), R^{n}\right)$ and $\{g(x(t), x([t]))\} \in \mathcal{L}^{2}\left([0, \infty), R^{n \times d}\right)$,

(3) equation (2.4) is satisfied on each interval $[n, n+1) \subset[0, \infty)$ with integral end-points almost surely. A solution $\{x(t)\}$ is said to be unique if any other solution $\{\bar{x}(t)\}$ is indistinguishable from $\{x(t)\}$, that is,

$$
P\{x(t)=\bar{x}(t) \forall t \in[0, \infty)\}=1 .
$$

Let $h=1 / m$ be a given stepsize with integer $m \geq 1$, and let the gridpoints $t_{n}$ be defined by $t_{n}=n h(n=0,1,2, \ldots)$. For simplicity, we assume that $T=N h$. We consider the EulerMaruyama method to (2.3),

$$
y_{n+1}=y_{n}+f\left(y_{n}, y^{h}([n h])\right) h+g\left(y_{n}, y^{h}([n h])\right) \Delta B_{n}
$$

for $n=0,1,2, \ldots$, where $\Delta B_{n}=B\left(t_{n}\right)-B\left(t_{n-1}\right), y^{h}([n h])$ is approximation to the exact solution $x([n h])$. Let $n=k m+l(k=0,1,2, \ldots, l=0,1,2, \ldots, m-1)$. The adaptation of the Euler method to (2.3) leads to a numerical process of the following type:

$$
y_{k m+l+1}=y_{k m+l}+f\left(y_{k m+l}, y_{k m}\right) h+g\left(y_{k m+l}, y_{k m}\right) \Delta B_{k m+l},
$$

where $\Delta B_{k m+l}=B\left(t_{k m+l}\right)-B\left(t_{k m+l-1}\right), y_{k m+l}$ and $y_{k m}$ are approximations to the exact solution $x\left(t_{k m+l}\right)$ and $x\left(\left[t_{k m+l}\right]\right)$, respectively. The continuous Euler-Maruyama approximate solution is defined by

$$
y(t)=y(0)+\int_{0}^{t} f(z(s), z([s])) d s+\int_{0}^{t} g(z(s), z([s])) d B(s)
$$

where $z(t)=y_{k m+l}$ and $z([t])=y_{k m}$ for $t \in\left[t_{k m+l}, t_{k m+l+1}\right)$. It is not difficult to see that $y\left(t_{k m+l}\right)=z\left(t_{k m+l}\right)=y_{k m+l}$ for $k=0,1,2, \ldots, l=0,1,2, \ldots, m-1$. For sufficiently large integer $i$, define the stopping times $\eta_{i}=\inf \{t \geq 0:|x(t)| \geq i\}, \theta_{i}=\inf \{t \geq 0:|y(t)| \geq i\}$. 


\section{Convergence in Probability of the Euler-Maruyama Method}

In this section, we concentrate on (2.3) under the local Lipschitz condition (H1) without the linear growth condition (H2) to establish the generalized existence and uniqueness theorem for stochastic differential equations with piecewise continuous arguments. We then give the convergence in probability of the EM method to (2.3) under the local Lipschitz condition (H1) and some additional conditions (H3).

Theorem 3.1. Under the conditions (H1) and (H3), there is a unique global solution $x(t)$ to (2.3) with initial data $x(0)=c_{0}$ on $t \in[0, \infty)$. Moreover, the solution has the property that

$$
E V(x(t))<\infty, \text { for any } t \geq 0 \text {. }
$$

Proof. Applying the standard truncation technique to (2.3), we obtain the unique maximal local solution $x(t)$ exists on $\left[0, \eta_{e}\right)$ under the local Lipschitz condition in a similar way as the proof of [10, Theorem 3.15, page 91], where $\eta_{e}$ is the explosion time. For each integer $i \geq\left|c_{0}\right|$, define the stopping time

$$
\eta_{i}=\inf \left\{t \in\left[0, \eta_{e}\right):|x(t)| \geq i\right\} .
$$

Clearly, $\eta_{i}$ is increasing as $i \rightarrow \infty$. We denote that $\eta_{\infty}=\lim _{i \rightarrow \infty} \eta_{i}$ and $\inf \emptyset=\infty$. Hence, $\eta_{\infty} \leq \eta_{e}$ almost surely. If we can obtain $\eta_{\infty}=\infty$ almost surely, then $\eta_{e}=\infty$ almost surely.

In what follows, we will prove $\eta_{\infty}=\infty$ almost surely and assertion (3.1). By the Itô formula and condition (2.8), we derive that

$$
\begin{aligned}
d V(x(t)) & =L V(x(t), x([t])) d t+V_{x}(x(t)) g(x(t), x([t])) d B(t) \\
& \leq \alpha[1+V(x(t))+V(x([t]))] d t+V_{x}(x(t)) g(x(t), x([t])) d B(t),
\end{aligned}
$$

for $0 \leq t<\eta_{\infty}$. Now, for $t_{1} \in[0,1)$, we can integrate both sides of (3.3) from 0 to $\eta_{i} \wedge t_{1}$,

$$
\begin{aligned}
V\left(x\left(\eta_{i} \wedge t_{1}\right)\right) \leq & V(x(0))+\alpha[1+V(x(0))] \\
& +\alpha \int_{0}^{\eta_{i} \wedge t_{1}} V(x(t)) d t+\int_{0}^{\eta_{i} \wedge t_{1}} V_{x}(x(t)) g(x(t), x([t])) d B(t) .
\end{aligned}
$$

We take the expectations in both sides of (3.4),

$$
\begin{aligned}
E V\left(x\left(\eta_{i} \wedge t_{1}\right)\right) & \leq V(x(0))+\alpha[1+V(x(0))]+\alpha E \int_{0}^{\eta_{i} \wedge t_{1}} V(x(t)) d t \\
& \leq \beta_{1}+\alpha E \int_{0}^{\eta_{i} \wedge t_{1}} V(x(t)) d t
\end{aligned}
$$

where

$$
\beta_{1}=V(x(0))+\alpha[1+V(x(0))]=(1+\alpha) V\left(c_{0}\right)+\alpha<\infty .
$$


It is easy to compute

$$
\begin{aligned}
E V\left(x\left(\eta_{i} \wedge t_{1}\right)\right) & \leq \beta_{1}+\alpha E \int_{0}^{\eta_{i} \wedge t_{1}} V(x(t)) d t \\
& =\beta_{1}+\alpha \int_{0}^{t_{1}} E V\left(x\left(\eta_{i} \wedge t\right)\right) d t
\end{aligned}
$$

Now the Gronwall inequality yields that

$$
E V\left(x\left(\eta_{i} \wedge t_{1}\right)\right) \leq \beta_{1} e^{\alpha t_{1}} \leq \beta_{1} e^{\alpha}, \quad 0 \leq t_{1}<1 .
$$

So we have

$$
E V\left(x\left(\eta_{i} \wedge 1\right)\right)=\lim _{t_{1} \rightarrow 1} E V\left(x\left(\eta_{i} \wedge t_{1}\right)\right) \leq \beta_{1} e^{\alpha} .
$$

Defining

$$
\gamma_{i}=\inf _{|x| \geq i} V(x), \quad \forall i \geq\left|c_{0}\right|
$$

denoting $I_{A}$ as the indicator function of a set $A$, we compute

$$
\beta_{1} e^{\alpha} \geq E V\left(x\left(\eta_{i} \wedge 1\right)\right) \geq E\left(V\left(x\left(\eta_{i}\right)\right) I_{\left\{\eta_{i} \leq 1\right\}}\right) \geq \gamma_{i} P\left(\eta_{i} \leq 1\right) .
$$

Letting $i \rightarrow \infty$, we have that $P\left(\eta_{\infty} \leq 1\right)=0$, namely,

$$
P\left(\eta_{\infty}>1\right)=1
$$

By (3.8) and (3.12),

$$
E V\left(x\left(t_{1}\right)\right) \leq \beta_{1} e^{\alpha}, \quad 0 \leq t_{1} \leq 1
$$

Now let us prove $\eta_{\infty}>2$, for $t_{2} \in[1,2)$, and we can integrate both sides of (3.3) from 1 to $\eta_{i} \wedge t_{2}$ and take the expectations

$$
\begin{aligned}
E V\left(x\left(\eta_{i} \wedge t_{2}\right)\right) & \leq E V(x(1))+\alpha[1+E V(x(1))]+\alpha E \int_{1}^{\eta_{i} \wedge t_{2}} V(x(t)) d t \\
& \leq \beta_{2}+\alpha \int_{1}^{t_{2}} E V\left(x\left(\eta_{i} \wedge t\right)\right) d t
\end{aligned}
$$

where

$$
\beta_{2} \leq \beta_{1} e^{\alpha}+\alpha\left(1+\beta_{1} e^{\alpha}\right)<\infty
$$


Now the Gronwall inequality yields that

$$
E V\left(x\left(\eta_{i} \wedge t_{2}\right)\right) \leq \beta_{2} e^{\alpha\left(t_{2}-1\right)} \leq \beta_{2} e^{\alpha}, \quad 1 \leq t_{2}<2 .
$$

Hence, we have

$$
E V\left(x\left(\eta_{i} \wedge 2\right)\right)=\lim _{t_{1} \rightarrow 2} E V\left(x\left(\eta_{i} \wedge t_{2}\right)\right) \leq \beta_{2} e^{\alpha} .
$$

By (3.10) and (3.17), we compute

$$
\beta_{2} e^{\alpha} \geq E V\left(x\left(\eta_{i} \wedge 2\right)\right) \geq E\left(V\left(x\left(\eta_{i}\right)\right) I_{\left\{\eta_{i} \leq 2\right\}}\right) \geq \gamma_{i} P\left(\eta_{i} \leq 2\right) .
$$

Letting $i \rightarrow \infty$, we have that $P\left(\eta_{\infty} \leq 2\right)=0$, namely,

$$
P\left(\eta_{\infty}>2\right)=1 \text {. }
$$

From (3.16) and (3.19), we yield

$$
E V\left(x\left(t_{2}\right)\right) \leq \beta_{2} e^{\alpha}, \quad 1 \leq t_{2} \leq 2 .
$$

Repeating this procedure, we can show that, for any integer $j \geq 1, \eta_{\infty}>j$ almost surely,

$$
E V\left(x\left(t_{j}\right)\right) \leq \beta_{j} e^{\alpha}, \quad j-1 \leq t_{j} \leq j,
$$

where

$$
\beta_{j} \leq \beta_{j-1} e^{\alpha}+\alpha\left(1+\beta_{j-1} e^{\alpha}\right)<\infty
$$

By (3.13), (3.20), and (3.21), we obtain

$$
E V(x(t)) \leq \beta_{j} e^{\alpha}<\infty, \quad 0 \leq t \leq j .
$$

Therefore, we must have $\eta_{\infty}=\infty$ almost surely as well as the required assertion (3.1). The proof is completed.

Theorem 3.2. Under the conditions (H1) and (H3), if $\varepsilon \in(0,1)$ and $T>0$, then there exists a sufficiently large integer $\hat{i}$, dependent on $\varepsilon$ and $T$ such that

$$
P\left(\eta_{i} \leq T\right) \leq \varepsilon, \quad \forall i \geq \hat{i}
$$

Proof. By Theorem 3.1, we have

$$
E V\left(x\left(\eta_{i} \wedge t\right)\right) \leq \beta_{j} e^{\alpha}<\infty, \quad 0 \leq t \leq j .
$$


Choose $j$ large enough for $j>T$. From (3.25), we get

$$
E V\left(x\left(\eta_{i} \wedge([T]+1)\right)\right) \leq \beta_{[T]+1} e^{\alpha}<\infty .
$$

It follows from (3.10) and (3.26) that

$$
\begin{aligned}
\beta_{[T]+1} e^{\alpha} & \geq E V\left(x\left(\eta_{i} \wedge([T]+1)\right)\right) \\
& \geq E\left(V\left(x\left(\eta_{i}\right)\right) I_{\left\{\eta_{i} \leq[T]+1\right\}}\right) \\
& \geq \gamma_{i} P\left(\eta_{i} \leq[T]+1\right),
\end{aligned}
$$

while by (H3), $\gamma_{i} \rightarrow \infty$ as $i \rightarrow \infty$. Thus, there is a sufficiently large integer $\hat{i}$ such that

$$
\gamma_{i} \geq \frac{\beta_{[T]+1} e^{\alpha}}{\varepsilon}, \quad \forall i \geq \widehat{i}
$$

Therefore, we get that

$$
P\left(\eta_{i} \leq T\right) \leq P\left(\eta_{i} \leq[T]+1\right) \leq \frac{\beta_{[T]+1} e^{\alpha}}{\gamma_{i}}<\varepsilon
$$

The proof is completed.

The following lemma shows that both $y(t)$ and $z(t)$ are close to each other.

Lemma 3.3. Under the condition (H1), let $T>0$ be arbitrary. Then

$$
E\left(\sup _{0 \leq t \leq T \wedge \theta_{i}}|y(t)-z(t)|^{2}\right) \leq C_{1}(i) h^{1 / 2}
$$

where $C_{1}(i)=4\left(2 i^{2} L_{i}+|f(0,0)|^{2} \vee|g(0,0)|^{2}\right)\left(1+(16 \sqrt{3} / 3) d(T+1)^{1 / 2}\right)$.

Proof. For $t \in\left[0, T \wedge \theta_{i}\right)$, there are two integers $k$ and $l$ such that $t \in\left[t_{k m+l}, t_{k m+l+1}\right)$. So we compute

$$
\begin{aligned}
|y(t)-z(t)|^{2} & =\left|\int_{t_{k m+l}}^{t} f(z(s), z([s])) d s+\int_{t_{k m+l}}^{t} g(z(s), z([s])) d B(s)\right|^{2} \\
& =\left|\int_{t_{k m+l}}^{t} f\left(y_{k m+l}, y_{k m}\right) d s+\int_{t_{k m+l}}^{t} g\left(y_{k m+l}, y_{k m}\right) d B(s)\right|^{2} \\
& =\left|f\left(y_{k m+l}, y_{k m}\right)\left(t-t_{k m+l}\right)+g\left(y_{k m+l}, y_{k m}\right)\left(B(t)-B\left(t_{k m+l}\right)\right)\right|^{2} \\
& \leq 2\left|f\left(y_{k m+l}, y_{k m}\right)\right|^{2} h^{2}+2\left|g\left(y_{k m+l}, y_{k m}\right)\right|^{2}\left|B(t)-B\left(t_{k m+l}\right)\right|^{2},
\end{aligned}
$$


Journal of Applied Mathematics

since

$$
\begin{aligned}
\left|f\left(y_{k m+l}, y_{k m}\right)\right|^{2} & \leq 2\left|f\left(y_{k m+l}, y_{k m}\right)-f(0,0)\right|^{2}+2|f(0,0)|^{2} \\
& \leq 2 L_{i}\left(\left|y_{k m+l}\right|^{2}+\left|y_{k m}\right|^{2}\right)+2|f(0,0)|^{2} \\
& \leq 2\left(2 i^{2} L_{i}+|f(0,0)|^{2}\right) .
\end{aligned}
$$

Similarly, we obtain that

$$
\left|g\left(y_{k m+l}, y_{k m}\right)\right|^{2} \leq 2\left(2 i^{2} L_{i}+|g(0,0)|^{2}\right)
$$

Substituting (3.32) and (3.33) into (3.31) gives

$$
|y(t)-z(t)|^{2} \leq C\left(h^{2}+\left|B(t)-B\left(t_{k m+l}\right)\right|^{2}\right),
$$

where $C=4\left(2 i^{2} L_{i}+|f(0,0)|^{2} \vee|g(0,0)|^{2}\right)$. Let $n_{t}=k m+l$ for $t \in\left[t_{k m+l}, t_{k m+l+1}\right)$, then we have that

$$
\begin{aligned}
E\left(\sup _{0 \leq t \leq T \wedge \theta_{i}}\left|B(t)-B\left(t_{n_{t}}\right)\right|^{2}\right) & \leq \sum_{i=1}^{d} E\left(\sup _{0 \leq t \leq T \wedge \theta_{i}}\left|B_{i}(t)-B_{i}\left(t_{n_{t}}\right)\right|^{2}\right) \\
& \leq \sum_{i=1}^{d} E\left(\sup _{u=0,1,2, \ldots, N} \sup _{t_{u} \leq t \leq t_{u+1} \wedge T}\left|B_{i}(t)-B_{i}\left(t_{u}\right)\right|^{2}\right) \\
& \leq \sum_{i=1}^{d}\left[E\left(\sup _{u=0,1,2, \ldots, N} \sup _{t_{u} \leq t \leq t_{u+1} \wedge T}\left|B_{i}(t)-B_{i}\left(t_{u}\right)\right|^{4}\right)\right]^{1 / 2},
\end{aligned}
$$

while by the Doob martingale inequality, we have

$$
\begin{aligned}
E\left(\sup _{u=0,1,2, \ldots, N} \sup _{t_{u} \leq t \leq t_{u+1} \wedge T}\left|B_{i}(t)-B_{i}\left(t_{u}\right)\right|^{4}\right) & \leq \sum_{u=0}^{N} E\left(\sup _{t_{u} \leq t \leq t_{u+1} \wedge T}\left|B_{i}(t)-B_{i}\left(t_{u}\right)\right|^{4}\right) \\
& \leq\left(\frac{4}{3}\right)^{4} \sum_{u=0}^{N} E\left|B_{i}\left(t_{u+1} \wedge T\right)-B_{i}\left(t_{u}\right)\right|^{4} \\
& \leq \frac{256}{27} \sum_{u=0}^{N} h^{2} \\
& \leq \frac{256}{27}(T+1) h .
\end{aligned}
$$


Substituting (3.36) into (3.35) yields

$$
E\left(\sup _{0 \leq t \leq T \wedge \theta_{i}}\left|B(t)-B\left(t_{n_{t}}\right)\right|^{2}\right) \leq \sum_{i=1}^{d}\left(\frac{256}{27}(T+1) h\right)^{1 / 2}=\frac{16 \sqrt{3}}{9} d(T+1)^{1 / 2} h^{1 / 2} .
$$

Thus, we obtain

$$
\begin{aligned}
E\left(\sup _{0 \leq t \leq T \wedge \theta_{i}}|y(t)-z(t)|^{2}\right) & \leq C h^{2}+C \frac{16 \sqrt{3}}{9} d(T+1)^{1 / 2} h^{1 / 2} \\
& \leq C\left(1+\frac{16 \sqrt{3}}{3} d(T+1)^{1 / 2}\right) h^{1 / 2} \\
& \leq C_{1}(i) h^{1 / 2},
\end{aligned}
$$

where $C_{1}(i)=4\left(2 i^{2} L_{i}+|f(0,0)|^{2} \vee|g(0,0)|^{2}\right)\left(1+(16 \sqrt{3} / 3) d(T+1)^{1 / 2}\right)$. The proof is completed.

Lemma 3.4. Under the condition (H1), for any $T>0$, there exists a positive constant $C_{2}(i)$ dependent on $i$ and independent of $h$ such that

$$
E\left(\sup _{0 \leq t \leq T}\left|x\left(t \wedge \eta_{i} \wedge \theta_{i}\right)-y\left(t \wedge \eta_{i} \wedge \theta_{i}\right)\right|^{2}\right) \leq C_{2}(i) h^{1 / 2}
$$

where $C_{2}(i)=8 T(T+4) L_{i} C_{1}(i) e^{8 T(T+4) L_{i}}$.

Proof. It follows from (2.4) and (2.12) that

$$
\begin{aligned}
\mid x(t & \left.\wedge \eta_{i} \wedge \theta_{i}\right)-\left.y\left(t \wedge \eta_{i} \wedge \theta_{i}\right)\right|^{2} \\
\leq & 2\left|\int_{0}^{t \wedge \eta_{i} \wedge \theta_{i}} f(x(s), x([s]))-f(z(s), z([s])) d s\right|^{2} \\
& +2\left|\int_{0}^{t \wedge \eta_{i} \wedge \theta_{i}} g(x(s), x([s]))-g(z(s), z([s])) d B(s)\right|^{2} .
\end{aligned}
$$


By the Hölder inequality, we obtain

$$
\begin{aligned}
\mid x(t \wedge & \left.\eta_{i} \wedge \theta_{i}\right)-\left.y\left(t \wedge \eta_{i} \wedge \theta_{i}\right)\right|^{2} \\
\leq & 2 T \int_{0}^{t}\left|f\left(x\left(s \wedge \eta_{i} \wedge \theta_{i}\right), x\left(\left[s \wedge \eta_{i} \wedge \theta_{i}\right]\right)\right)-f\left(z\left(s \wedge \eta_{i} \wedge \theta_{i}\right), z\left(\left[s \wedge \eta_{i} \wedge \theta_{i}\right]\right)\right)\right|^{2} d s \\
& +2\left|\int_{0}^{t} g\left(x\left(s \wedge \eta_{i} \wedge \theta_{i}\right), x\left(\left[s \wedge \eta_{i} \wedge \theta_{i}\right]\right)\right)-g\left(z\left(s \wedge \eta_{i} \wedge \theta_{i}\right), z\left(\left[s \wedge \eta_{i} \wedge \theta_{i}\right]\right)\right) d B(s)\right|^{2} .
\end{aligned}
$$

This implies that for any $0 \leq t_{1} \leq T$,

$$
\begin{aligned}
& E \sup _{0 \leq t \leq t_{1}}\left(\left|x\left(t \wedge \eta_{i} \wedge \theta_{i}\right)-y\left(t \wedge \eta_{i} \wedge \theta_{i}\right)\right|^{2}\right) \\
& \leq 2 T E \sup _{0 \leq t \leq t_{1}} \int_{0}^{t} \mid f\left(x\left(s \wedge \eta_{i} \wedge \theta_{i}\right), x\left(\left[s \wedge \eta_{i} \wedge \theta_{i}\right]\right)\right) \\
& \quad-\left.f\left(z\left(s \wedge \eta_{i} \wedge \theta_{i}\right), z\left(\left[s \wedge \eta_{i} \wedge \theta_{i}\right]\right)\right)\right|^{2} d s \\
& +2 E\left[\sup _{0 \leq t \leq t_{1}} \mid \int_{0}^{t} g\left(x\left(s \wedge \eta_{i} \wedge \theta_{i}\right), x\left(\left[s \wedge \eta_{i} \wedge \theta_{i}\right]\right)\right)\right. \\
& \left.\quad-\left.g\left(z\left(s \wedge \eta_{i} \wedge \theta_{i}\right), z\left(\left[s \wedge \eta_{i} \wedge \theta_{i}\right]\right)\right) d B(s)\right|^{2}\right] .
\end{aligned}
$$

By Doob martingale inequality, it is not difficult to show that

$$
\begin{aligned}
& E \sup _{0 \leq t \leq t_{1}}\left(\left|x\left(t \wedge \eta_{i} \wedge \theta_{i}\right)-y\left(t \wedge \eta_{i} \wedge \theta_{i}\right)\right|^{2}\right) \\
& \leq 2 T E \int_{0}^{t_{1}}\left|f\left(x\left(s \wedge \eta_{i} \wedge \theta_{i}\right), x\left(\left[s \wedge \eta_{i} \wedge \theta_{i}\right]\right)\right)-f\left(z\left(s \wedge \eta_{i} \wedge \theta_{i}\right), z\left(\left[s \wedge \eta_{i} \wedge \theta_{i}\right]\right)\right)\right|^{2} d s \\
&+8 E \int_{0}^{t_{1}}\left|g\left(x\left(s \wedge \eta_{i} \wedge \theta_{i}\right), x\left(\left[s \wedge \eta_{i} \wedge \theta_{i}\right]\right)\right)-g\left(z\left(s \wedge \eta_{i} \wedge \theta_{i}\right), z\left(\left[s \wedge \eta_{i} \wedge \theta_{i}\right]\right)\right)\right|^{2} d s .
\end{aligned}
$$


Note from (H1) and Lemma 3.3 that

$$
\begin{aligned}
E \int_{0}^{t_{1}}\left|f\left(x\left(s \wedge \eta_{i} \wedge \theta_{i}\right), x\left(\left[s \wedge \eta_{i} \wedge \theta_{i}\right]\right)\right)-f\left(z\left(s \wedge \eta_{i} \wedge \theta_{i}\right), z\left(\left[s \wedge \eta_{i} \wedge \theta_{i}\right]\right)\right)\right|^{2} d s \\
\leq L_{i} E \int_{0}^{t_{1}}\left|x\left(s \wedge \eta_{i} \wedge \theta_{i}\right)-z\left(s \wedge \eta_{i} \wedge \theta_{i}\right)\right|^{2}+\left|x\left(\left[s \wedge \eta_{i} \wedge \theta_{i}\right]\right)-z\left(\left[s \wedge \eta_{i} \wedge \theta_{i}\right]\right)\right|^{2} d s \\
\leq 2 L_{i} E \int_{0}^{t_{1}}\left|x\left(s \wedge \eta_{i} \wedge \theta_{i}\right)-y\left(s \wedge \eta_{i} \wedge \theta_{i}\right)\right|^{2} d s \\
\quad+2 L_{i} E \int_{0}^{t_{1}}\left|y\left(s \wedge \eta_{i} \wedge \theta_{i}\right)-z\left(s \wedge \eta_{i} \wedge \theta_{i}\right)\right|^{2} d s \\
\quad+2 L_{i} E \int_{0}^{t_{1}}\left|x\left(\left[s \wedge \eta_{i} \wedge \theta_{i}\right]\right)-y\left(\left[s \wedge \eta_{i} \wedge \theta_{i}\right]\right)\right|^{2} d s \\
\quad+2 L_{i} E \int_{0}^{t_{1}}\left|y\left(\left[s \wedge \eta_{i} \wedge \theta_{i}\right]\right)-z\left(\left[s \wedge \eta_{i} \wedge \theta_{i}\right]\right)\right|^{2} d s \\
\leq 4 L_{i} \int_{0}^{t_{1}} E \sup _{0 \leq t \leq s \wedge \eta_{i} \wedge \theta_{i}}|x(t)-y(t)|^{2} d s+4 L_{i} T C_{1}(i) h^{1 / 2} .
\end{aligned}
$$

Similarly, we obtain that

$$
\begin{aligned}
& E \int_{0}^{t_{1}}\left|g\left(x\left(s \wedge \eta_{i} \wedge \theta_{i}\right), x\left(\left[s \wedge \eta_{i} \wedge \theta_{i}\right]\right)\right)-g\left(z\left(s \wedge \eta_{i} \wedge \theta_{i}\right), z\left(\left[s \wedge \eta_{i} \wedge \theta_{i}\right]\right)\right)\right|^{2} d s \\
& \quad \leq 4 L_{i} \int_{0}^{t_{1}} E \sup _{0 \leq t \leq s \wedge \eta_{i} \wedge \theta_{i}}|x(t)-y(t)|^{2} d s+4 L_{i} T C_{1}(i) h^{1 / 2}
\end{aligned}
$$

Substituting (3.44) and (3.45) into (3.43) gives

$$
\begin{aligned}
& E \sup _{0 \leq t \leq t_{1}}\left(\left|x\left(t \wedge \eta_{i} \wedge \theta_{i}\right)-y\left(t \wedge \eta_{i} \wedge \theta_{i}\right)\right|^{2}\right) \\
& \quad \leq 8(T+4) L_{i} \int_{0}^{t_{1}} E \sup _{0 \leq t \leq s \wedge \eta_{i} \wedge \theta_{i}}|x(t)-y(t)|^{2} d s+8(T+4) L_{i} T C_{1}(i) h^{1 / 2}
\end{aligned}
$$

By the Gronwall inequality, we must get

$$
E \sup _{0 \leq t \leq T}\left(\left|x\left(t \wedge \eta_{i} \wedge \theta_{i}\right)-y\left(t \wedge \eta_{i} \wedge \theta_{i}\right)\right|^{2}\right) \leq C_{2}(i) h^{1 / 2},
$$

where $C_{2}(i)=8 T(T+4) L_{i} C(1+4 d) e^{8 T(T+4) L_{i}}$. 
Journal of Applied Mathematics

Lemma 3.5. Under the conditions (H1) and (H3) if $\varepsilon \in(0,1)$ and $T>0$, then there exists a sufficiently large integer $\hat{i}$ (dependent on $\varepsilon$ and $T$ ) and sufficiently small $\hat{h}$ such that

$$
P\left(\theta_{\hat{i}} \leq T\right) \leq \varepsilon \quad \forall h \leq \widehat{h} .
$$

Proof. By Itô formula, we have

$$
\begin{aligned}
d V(y(t))= & \left(V_{y}(y(t)) f(z(t), z([t]))+\frac{1}{2} \operatorname{trace}\left[g^{T}(z(t), z([t])) V_{y y}(y(t)) g(z(t), z([t]))\right]\right) d t \\
& +V_{y}(y(t)) g(z(t), z([t])) d B(t) \\
= & \left(L V(y(t), y([t]))+V_{y}(y(t))[f(z(t), z([t]))-f(y(t), y([t]))]\right. \\
& +\frac{1}{2} \operatorname{trace}\left[g^{T}(z(t), z([t])) V_{y y}(y(t)) g(z(t), z([t]))\right. \\
& \left.\left.\quad-g^{T}(y(t), y([t])) V_{y y}(y(t)) g(y(t), y([t]))\right]\right) d t \\
& +V_{y}(y(t)) g(z(t), z([t])) d B(t) .
\end{aligned}
$$

By condition (H1),

$$
\begin{aligned}
& V_{y}(y(t)) {[f(z(t), z([t]))-f(y(t), y([t]))] } \\
&+ \frac{1}{2} \operatorname{trace}\left[g^{T}(z(t), z([t])) V_{y y}(y(t)) g(z(t), z([t]))-g^{T}(y(t), y([t])) V_{y y}(y(t)) g(y(t), y([t]))\right] \\
&=\left(V_{y}(y(s))[f(z(s), z([s]))-f(y(s), y([s]))]\right. \\
& \quad+\frac{1}{2} \operatorname{trace}\left(\left[g^{T}(z(s), z([s]))-g^{T}(y(s), y([s]))\right] V_{y y}(y(s)) g(z(s), z([s]))\right. \\
& \quad+\frac{1}{2} \operatorname{trace}\left(g^{T}(y(s), y([s])) V_{y y}(y(s))[g(z(s), z([s]))-g(y(s), y([s]))]\right. \\
& \leq c_{i}(|y(t)-z(t)|+|y([t])-z([t])|),
\end{aligned}
$$

where $c_{i}$ denotes a positive constant independent of $h$. Substituting (3.50) into (3.49), we obtain that, for $0 \leq t \leq \theta_{i}$,

$$
\begin{aligned}
d V(y(t)) \leq & \left(L V(y(t), y([t]))+c_{i}(|y(t)-z(t)|+|y([t])-z([t])|)\right) d t \\
& +V_{y}(y(t)) g(z(t), z([t])) d B(t) .
\end{aligned}
$$


Hence, for $t \in[n, n+1)$, we can integrate both sides of (3.51) from $n$ to $t \wedge \theta_{i}$ and take the expectations

$$
\begin{aligned}
E V\left(y\left(t \wedge \theta_{i}\right)\right) \leq & E V(y(n))+E \int_{n}^{t \wedge \theta_{i}} L V(y(s), y([s])) d s \\
& +E \int_{n}^{t \wedge \theta_{i}} c_{i}(|y(t)-z(t)|+|y([t])-z([t])|) d s,
\end{aligned}
$$

while

$$
\begin{aligned}
& c_{i} E \int_{n}^{t}\left(\left|y\left(s \wedge \theta_{i}\right)-z\left(s \wedge \theta_{i}\right)\right|+\left|y\left(\left[s \wedge \theta_{i}\right]\right)-z\left(\left[s \wedge \theta_{i}\right]\right)\right|\right) d s \\
& \quad=c_{i} \int_{n}^{t} E\left|y\left(s \wedge \theta_{i}\right)-z\left(s \wedge \theta_{i}\right)\right| d s+c_{i} \int_{n}^{t} E\left|y\left(\left[s \wedge \theta_{i}\right]\right)-z\left(\left[s \wedge \theta_{i}\right]\right)\right| d s \\
& \quad \leq c_{i} \int_{n}^{t}\left(E\left|y\left(s \wedge \theta_{i}\right)-z\left(s \wedge \theta_{i}\right)\right|^{2}\right)^{1 / 2} d s+c_{i} \int_{n}^{t}\left(E\left|y\left(\left[s \wedge \theta_{i}\right]\right)-z\left(\left[s \wedge \theta_{i}\right]\right)\right|^{2}\right)^{1 / 2} d s \\
& \quad \leq 2 c_{i} \int_{n}^{t}\left(E \sup _{0 \leq u \leq s \wedge \theta_{i}}|y(u)-z(u)|^{2}\right)^{1 / 2} d s \\
& \quad \leq 2 c_{i} \int_{n}^{t}\left[C_{1}(i) h^{1 / 2}\right]^{1 / 2} d s \\
& \quad \leq 2 c_{i} T\left[C_{1}(i) h^{1 / 2}\right]^{1 / 2} \\
& \quad \leq C_{3}(i) h^{1 / 4}
\end{aligned}
$$

where $C_{3}(i)=2 c_{i} T\left(C_{1}(i)\right)^{1 / 2}$. Substituting this into (3.52) yields that

$$
E V\left(y\left(t \wedge \theta_{i}\right)\right) \leq E V(y(n))+\tilde{\beta}+E \int_{n}^{t \wedge \theta_{i}}(L V(y(s), y([s]))) d s,
$$

where $\tilde{\beta}=C_{3}(i) h^{1 / 4}$. For $t \in[0,1)$, by condition (H3), we obtain that

$$
\begin{aligned}
E V\left(y\left(t \wedge \theta_{i}\right)\right) & \leq V(y(0))+\alpha E \int_{0}^{t \wedge \theta_{i}}[1+V(y(s))+V(y([s]))] d s+\tilde{\beta} \\
& \leq V(y(0))+\alpha[1+V(y(0))]+\tilde{\beta}+\alpha E \int_{0}^{t \wedge \theta_{i}} V(y(s)) d s \\
& \leq \tilde{\beta}+\widehat{\beta}_{1}+\alpha \int_{0}^{t} E V\left(y\left(s \wedge \theta_{i}\right)\right) d s
\end{aligned}
$$


Journal of Applied Mathematics

where

$$
\widehat{\beta}_{1}=V(y(0))+\alpha[1+V(y(0))]=(1+\alpha) V\left(c_{0}\right)+\alpha<\infty .
$$

Hence, by the Gronwall inequality,

$$
\begin{aligned}
E V\left(y\left(t \wedge \theta_{i}\right)\right) & \leq \tilde{\beta}+\widehat{\beta}_{1}+\alpha \int_{0}^{t} E V\left(y\left(s \wedge \theta_{i}\right)\right) d s \\
& \leq\left(\tilde{\beta}+\widehat{\beta}_{1}\right) e^{\alpha t} \\
& \leq\left(\tilde{\beta}+\widehat{\beta}_{1}\right) e^{\alpha} \\
& <\infty
\end{aligned}
$$

for $0 \leq t<1$. Consequently,

$$
E V\left(y\left(1 \wedge \theta_{i}\right)\right)=\lim _{t \rightarrow 1} E V\left(y\left(t \wedge \theta_{i}\right)\right) \leq\left(\tilde{\beta}+\widehat{\beta}_{1}\right) e^{\alpha}<\infty .
$$

Define

$$
\gamma_{i}=\inf _{|y| \geq i} V(y), \quad \forall i \geq\left|c_{0}\right|
$$

and denote $I_{A}$ as the indicator function of a set $A$, then we have

$$
\left(\tilde{\beta}+\widehat{\beta}_{1}\right) e^{\alpha} \geq E V\left(y\left(\theta_{i} \wedge 1\right)\right) \geq E\left(V\left(y\left(\theta_{i}\right)\right) I_{\left\{\theta_{i} \leq 1\right\}}\right) \geq \gamma_{i} P\left(\theta_{i} \leq 1\right) .
$$

Letting $i \rightarrow \infty$, we have that $P\left(\theta_{\infty} \leq 1\right)=0$, namely,

$$
P\left(\theta_{\infty}>1\right)=1
$$

By (3.57) and (3.61),

$$
E V(y(t)) \leq\left(\tilde{\beta}+\widehat{\beta}_{1}\right) e^{\alpha}<\infty, \quad 0 \leq t \leq 1
$$

For $t \in[1,2)$, by (3.54), we have

$$
\begin{aligned}
E V\left(y\left(t \wedge \theta_{i}\right)\right) & \leq E V(y(1))+\alpha[1+E V(y(1))]+\tilde{\beta}+\alpha \int_{1}^{t} E V\left(y\left(s \wedge \theta_{i}\right)\right) d s \\
& \leq \tilde{\beta}+\widehat{\beta}_{2}+\alpha \int_{1}^{t} E V\left(y\left(s \wedge \theta_{i}\right)\right) d s,
\end{aligned}
$$


where

$$
\widehat{\beta}_{2} \leq(1+\alpha)\left(\tilde{\beta}+\widehat{\beta}_{1}\right) e^{\alpha}+\alpha<\infty
$$

Hence, by the Gronwall inequality,

$$
\begin{aligned}
E V\left(y\left(t \wedge \theta_{i}\right)\right) & \leq \tilde{\beta}+\widehat{\beta}_{2}+\alpha \int_{1}^{t} E V\left(y\left(s \wedge \theta_{i}\right)\right) d s \\
& \leq\left(\tilde{\beta}+\widehat{\beta}_{2}\right) e^{\alpha(t-1)} \\
& \leq\left(\tilde{\beta}+\widehat{\beta}_{2}\right) e^{\alpha} \\
& <\infty
\end{aligned}
$$

for $1 \leq t<2$. Consequently, we can obtain that

$$
E V\left(y\left(2 \wedge \theta_{i}\right)\right)=\lim _{t \rightarrow 2} E V\left(y\left(t \wedge \theta_{i}\right)\right) \leq\left(\widetilde{\beta}+\widehat{\beta}_{2}\right) e^{\alpha}<\infty .
$$

In the same way, we have

$$
E V(y(t)) \leq\left(\tilde{\beta}+\widehat{\beta}_{2}\right) e^{\alpha}<\infty, \quad 1 \leq t \leq 2
$$

Repeating this procedure, for $t \in[N-1, T)$, we can show that

$$
E V\left(y\left(t \wedge \theta_{i}\right)\right) \leq\left(\widetilde{\beta}+\widehat{\beta}_{T}\right) e^{\alpha}<\infty,
$$

where

$$
\widehat{\beta}_{T} \leq(1+\alpha)\left(\tilde{\beta}+\widehat{\beta}_{N-1}\right) e^{\alpha}+\alpha<\infty .
$$

Consequently, we can obtain that

$$
E V\left(y\left(T \wedge \theta_{i}\right)\right)=\lim _{t \rightarrow T} E V\left(y\left(t \wedge \theta_{i}\right)\right) \leq\left(\tilde{\beta}+\widehat{\beta}_{T}\right) e^{\alpha} .
$$

We compute

$$
\left(\tilde{\beta}+\widehat{\beta}_{T}\right) e^{\alpha} \geq E V\left(y\left(T \wedge \theta_{i}\right)\right) \geq E\left(V\left(y\left(\theta_{i}\right)\right) I_{\left\{\theta_{i} \leq T\right\}}\right) \geq \gamma_{i} P\left(\theta_{i} \leq T\right) .
$$


Then we have

$$
P\left(\theta_{i} \leq T\right) \leq \frac{\left(\tilde{\beta}+\widehat{\beta}_{T}\right) e^{\alpha}}{\gamma_{i}}=\frac{\left(C_{3}(i) h^{1 / 4}+\widehat{\beta}_{T}\right) e^{\alpha}}{\gamma_{i}}
$$

Now, for any $\varepsilon \in(0,1)$, choose $i=\hat{i}$ sufficiently large for

$$
\frac{\widehat{\beta}_{T} e^{\alpha}}{\gamma_{i}} \leq \frac{\varepsilon}{2}
$$

and then choose $\widehat{h}$ sufficiently small for

$$
\frac{C_{3}(\hat{i}) \hat{h}^{1 / 4} e^{\alpha}}{r_{\hat{i}}} \leq \frac{\varepsilon}{2} .
$$

Hence,

$$
P\left(\theta_{\hat{i}} \leq T\right) \leq \varepsilon \quad \forall h \leq \widehat{h}
$$

The following theorems describe the convergence in probability of the EM method to (2.3) under the local Lipschitz condition (H1) and some additional conditions (H3).

Theorem 3.6. Under the conditions (H1) and (H3), for arbitrarily small $\sigma \in(0,1)$,

$$
\lim _{h \rightarrow 0} P\left(\omega: \sup _{0 \leq t \leq T}|x(t)-y(t)|>\sigma\right)=0,
$$

for any $T>0$.

Proof. For arbitrarily small $\sigma, \varepsilon \in(0,1)$. We set

$$
\bar{\Omega}=\left\{\omega: \sup _{0 \leq t \leq T}|x(t)-y(t)|>\sigma\right\}
$$

By Theorem 3.2 and Lemma 3.5, there exists a pair of $\widehat{i}$ and $\widehat{h}$ such that

$$
\begin{gathered}
P\left(\eta_{i} \leq T\right) \leq \frac{\varepsilon}{3}, \\
P\left(\theta_{\hat{i}} \leq T\right) \leq \frac{\varepsilon}{3}, \quad \forall h \leq \widehat{h} .
\end{gathered}
$$


For $h \leq \widehat{h}$,

$$
\begin{aligned}
P(\bar{\Omega}) & \leq P\left(\bar{\Omega} \cap\left\{\eta_{\hat{i}} \wedge \theta_{\hat{i}}>T\right\}\right)+P\left(\eta_{\hat{i}} \wedge \theta_{\hat{i}} \leq T\right) \\
& \leq P\left(\bar{\Omega} \cap\left\{\eta_{\hat{i}} \wedge \theta_{\hat{i}}>T\right\}\right)+P\left(\eta_{\hat{i}} \leq T\right)+P\left(\theta_{\hat{i}} \leq T\right) \\
& \leq P\left(\bar{\Omega} \cap\left\{\eta_{\hat{i}} \wedge \theta_{\hat{i}}>T\right\}\right)+\frac{2 \varepsilon}{3} .
\end{aligned}
$$

By Lemma 3.4, we get

$$
\begin{aligned}
\sigma^{2} P\left(\bar{\Omega} \cap\left\{\eta_{\hat{i}} \wedge \theta_{\hat{i}}>T\right\}\right) & \leq E\left[\sup _{0 \leq t \leq T}\left|x\left(t \wedge \eta_{\hat{i}} \wedge \theta_{\hat{i}}\right)-y\left(t \wedge \eta_{\hat{i}} \wedge \theta_{\hat{i}}\right)\right|^{2} I_{\left\{\eta_{\hat{i}} \wedge \theta_{\hat{i}}>T\right\}}\right] \\
& \leq E \sup _{0 \leq t \leq T}\left|x\left(t \wedge \eta_{\hat{i}} \wedge \theta_{\hat{i}}\right)-y\left(t \wedge \eta_{\hat{i}} \wedge \theta_{\hat{i}}\right)\right|^{2} \\
& \leq C_{2}(\widehat{i}) h^{1 / 2} .
\end{aligned}
$$

Hence,

$$
P\left(\bar{\Omega} \cap\left\{\eta_{\hat{i}} \wedge \theta_{\hat{i}}>T\right\}\right) \leq \frac{C_{2}(\hat{i}) h^{1 / 2}}{\sigma^{2}} .
$$

For all sufficiently small $h$, we obtain

$$
P\left(\bar{\Omega} \cap\left\{\eta_{i} \wedge \theta_{\hat{i}}>T\right\}\right) \leq \frac{\varepsilon}{3} .
$$

From (3.79) and (3.82), we see that for all sufficiently small $h$,

$$
P(\bar{\Omega}) \leq \varepsilon
$$

which proves the theorem.

Of course, $z(t)$ is computable but $y(t)$ is not, so the following theorem is much more useful in practice.

Theorem 3.7. Under the conditions (H1) and (H3), for arbitrarily small $\sigma \in(0,1)$,

$$
\lim _{h \rightarrow 0} P\left(\omega: \sup _{0 \leq t \leq T}|x(t)-z(t)|>\sigma\right)=0
$$

for any $T>0$. 
Journal of Applied Mathematics

Proof. For arbitrarily small $\sigma, \varepsilon \in(0,1)$. We denote

$$
\widehat{\Omega}=\left\{\omega: \sup _{0 \leq t \leq T}|x(t)-z(t)|>\sigma\right\} .
$$

In the same way as Theorem 3.6, we can see that

$$
P(\widehat{\Omega}) \leq P\left(\widehat{\Omega} \cap\left\{\eta_{\hat{i}} \wedge \theta_{\hat{i}}>T\right\}\right)+\frac{2 \varepsilon}{3} .
$$

But by Lemma 3.3, we get

$$
\begin{aligned}
\sigma^{2} P\left(\widehat{\Omega} \cap\left\{\eta_{\hat{i}} \wedge \theta_{\hat{i}}>T\right\}\right) \leq & E\left[\sup _{0 \leq t \leq T}\left|x\left(t \wedge \eta_{\hat{i}} \wedge \theta_{\hat{i}}\right)-z\left(t \wedge \eta_{\hat{i}} \wedge \theta_{\hat{i}}\right)\right|^{2} I_{\left\{\eta_{i} \wedge \theta_{i}>T\right\}}\right] \\
\leq & E \sup _{0 \leq t \leq T}\left|x\left(t \wedge \eta_{\hat{i}} \wedge \theta_{\hat{i}}\right)-z\left(t \wedge \eta_{\hat{i}} \wedge \theta_{\hat{i}}\right)\right|^{2} \\
\leq & 2 E \sup _{0 \leq t \leq T}\left|x\left(t \wedge \eta_{\hat{i}} \wedge \theta_{\hat{i}}\right)-y\left(t \wedge \eta_{\hat{i}} \wedge \theta_{\hat{i}}\right)\right|^{2} \\
& +2 E \sup _{0 \leq t \leq T}\left|y\left(t \wedge \eta_{\hat{i}} \wedge \theta_{\hat{i}}\right)-z\left(t \wedge \eta_{\hat{i}} \wedge \theta_{\hat{i}}\right)\right|^{2} \\
\leq & 2 E \sup _{0 \leq t \leq T}\left|x\left(t \wedge \eta_{\hat{i}} \wedge \theta_{\hat{i}}\right)-y\left(t \wedge \eta_{\hat{i}} \wedge \theta_{\hat{i}}\right)\right|^{2} \\
& +2 E \sup _{0 \leq t \leq T}\left|y\left(t \wedge \theta_{\hat{i}}\right)-z\left(t \wedge \theta_{\hat{i}}\right)\right|^{2} \\
\leq & 2\left(C_{2}(\widehat{i})+C_{1}(\hat{i})\right) h^{1 / 2} .
\end{aligned}
$$

therefore,

$$
P\left(\widehat{\Omega} \cap\left\{\eta_{\hat{i}} \wedge \theta_{\hat{i}}>T\right\}\right) \leq \frac{2\left(C_{2}(\hat{i})+C_{1}(\hat{i})\right) h^{1 / 2}}{\sigma^{2}} .
$$

For all sufficiently small $h$, we obtain

$$
P\left(\widehat{\Omega} \cap\left\{\eta_{\hat{i}} \wedge \theta_{\widehat{i}}>T\right\}\right) \leq \frac{\varepsilon}{3} .
$$

From (3.86) and (3.89), we see that for all sufficiently small $h$,

$$
P(\widehat{\Omega}) \leq \varepsilon,
$$

which proves the assertion (3.84). 


\section{Numerical Example}

Let us now discuss a numerical example to demonstrate the results which we obtain.

Example 4.1. Let us consider the stochastic differential equations with piecewise continuous arguments

$$
d x(t)=\left[-x^{3}(t)+x([t])\right] d t+\left[\sin x^{2}(t)+x([t])\right] d B(t) \quad \forall t \geq 0 .
$$

Defining $V(x)=x^{2}$, we have

$$
L V(x, y)=2 x\left(-x^{3}+y\right)+\left(\sin x^{2}+y\right)^{2} \leq-2 x^{4}+2 x y+2\left(\sin x^{2}\right)^{2}+2 y^{2} \leq 3\left(1+x^{2}+y^{2}\right)
$$

where $\alpha=3$. In other words, the equation satisfies condition (H3). By Theorem 3.1, we can conclude that the SEPCA (4.1) has a unique global solution $x(t)$ on $t \in[0, \infty)$. Moreover, the EM method can be applied to approximate the solution of the SEPCA (4.1). Given the stepsize $h=1 / m$, by (2.10), (2.11), and (2.12), the Euler method to (4.1) leads to a numerical process of the following type:

$$
y_{k m+l+1}=y_{k m+l}+\left(-y_{k m+l}^{3}+y_{k m}\right) h+\left(\sin y_{k m+l}^{2}+y_{k m}\right) \Delta B_{k m+l}
$$

The continuous Euler-Maruyama approximate solution is defined by

$$
y(t)=y(0)+\int_{0}^{t}\left(-z^{3}(s)+z([s])\right) d s+\int_{0}^{t}\left(\sin z^{2}(s)+z([s])\right) d B(s)
$$

where $z(t)=y_{k m+l}$ and $z([t])=y_{k m}$ for $t \in\left[t_{k m+l}, t_{k m+l+1}\right)$. By Theorems 3.6 and 3.7, we also have the convergence in probability of the EM method to (4.1) under the local Lipschitz condition (H1) and some additional conditions (H3).

\section{Acknowledgment}

The financial support from the National Natural Science Foundation of China (no. 11071050) is gratefully acknowledged.

\section{References}

[1] A. Friedman, Differential Equations and Applications. Volume 1 and 2, Academic Press, New York, NY, USA, 1975.

[2] R. Z. Khasminskii, Stochastic Stability of Differential Equations, Sijthoff and Noordhoff, Groningen, The Netherlands, 1980, Russian Translation Nauka, Moscow, Russia, 1969.

[3] X. Mao, Stability of Stochastic Differential Equations with Respect to Semimartingales, Long-man Scientific and Technical, London, UK, 1994. 
[4] X. Mao, Exponential Stability of Stochastic Differential Equations, Marcel Dekker, New York, NY, USA, 1994.

[5] X. Mao, Stochastic Differential Equations and Applications, Horwood Publishing Limited, Chichester, UK, 1997.

[6] X. Mao, Stochastic Differential Equations with Markovian Switching, Imperial College Press, London, UK, 2006.

[7] X. Mao, "A note on the LaSalle-type theorems for stochastic differential delay equations," Journal of Mathematical Analysis and Applications, vol. 268, no. 1, pp. 125-142, 2002.

[8] K. L. Cooke and J. Wiener, "Retarded differential equations with piecewise constant delays," Journal of Mathematical Analysis and Applications, vol. 99, no. 1, pp. 265-297, 1984.

[9] J. Wiener, Generalized Solutions of Functional Differential Equations, World scientific, Singapore, 1993.

[10] X. Mao, "Numerical solutions of stochastic differential delay equations under the generalized Khasminskii-type conditions," Applied Mathematics and Computation, vol. 217, no. 12, pp. 5512-5524, 2011.

[11] H. Y. Dai and M. Z. Liu, "Mean square stability of stochastic differential equations with piecewise continuous arguments," Journal of Natural Science of Heilongjiang University, vol. 25, no. 5, pp. 625-629, 2008. 


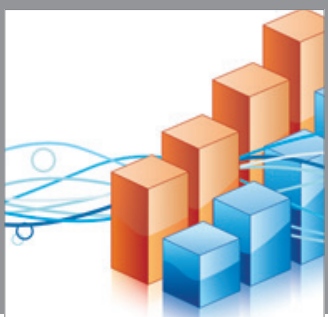

Advances in

Operations Research

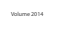

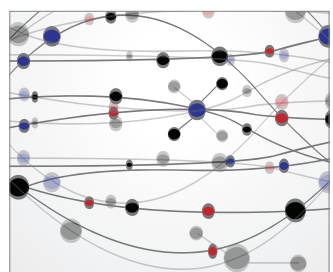

\section{The Scientific} World Journal
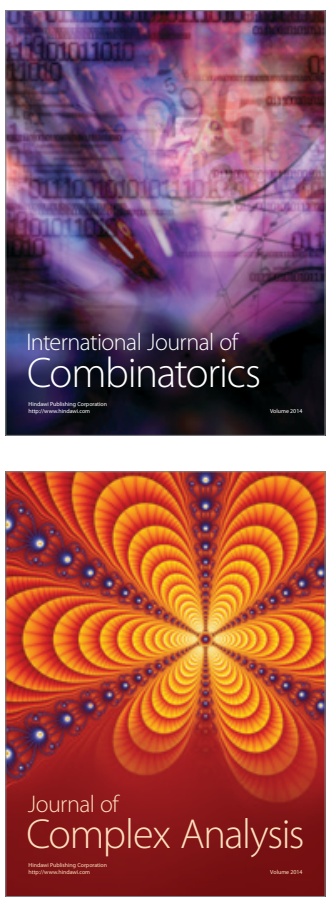

International Journal of

Mathematics and

Mathematical

Sciences
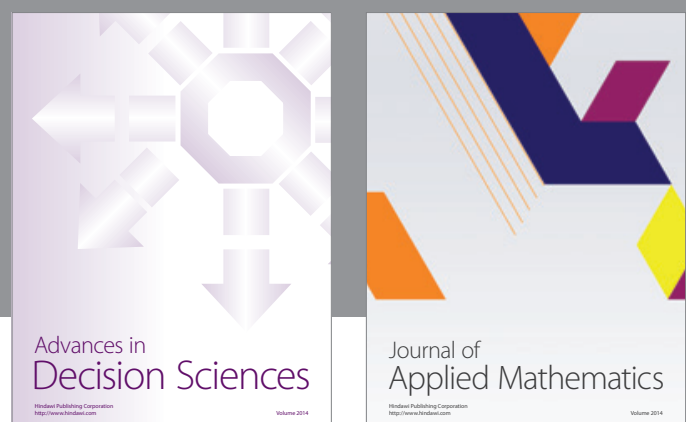

Journal of

Applied Mathematics
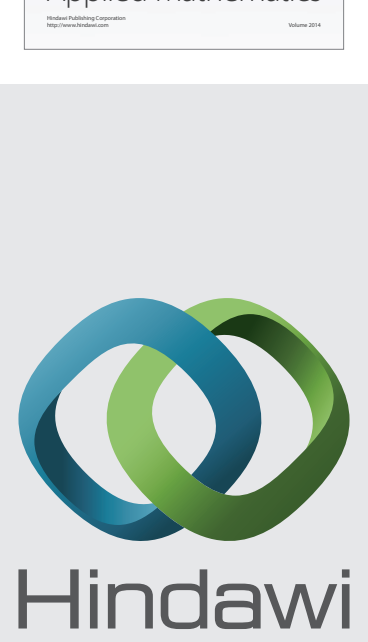

Submit your manuscripts at http://www.hindawi.com
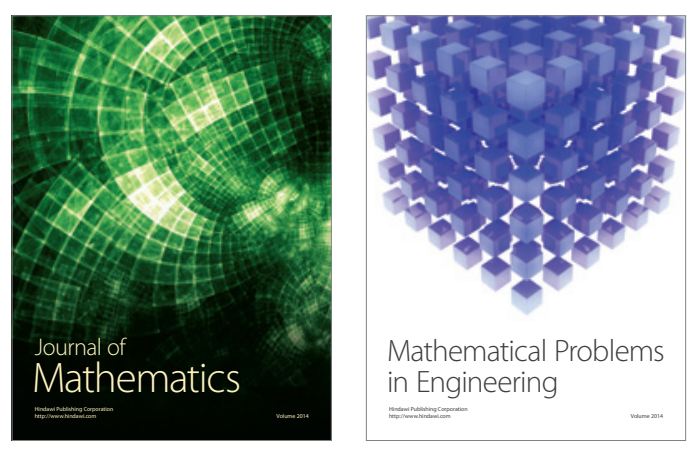

Mathematical Problems in Engineering
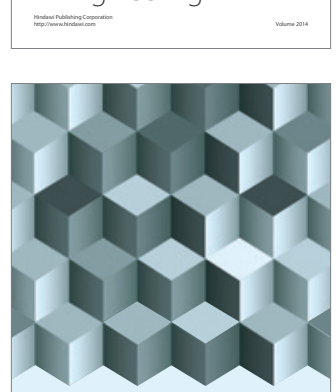

Journal of

Function Spaces
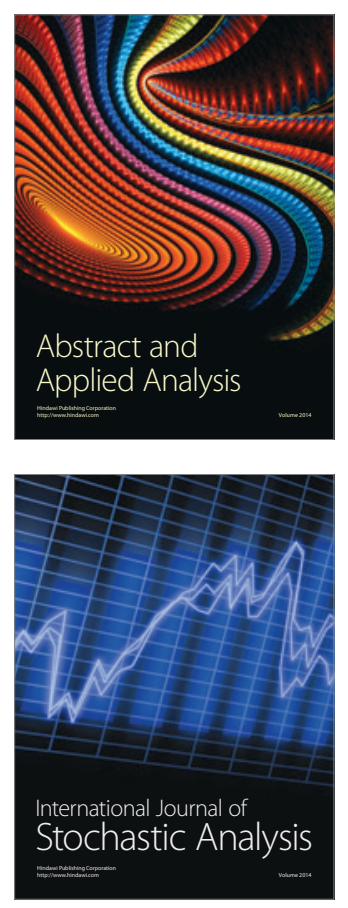

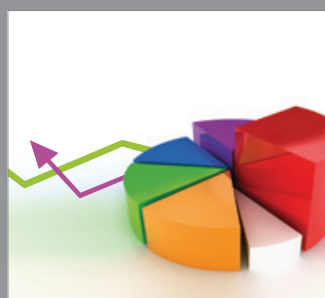

ournal of

Probability and Statistics

Promensencen
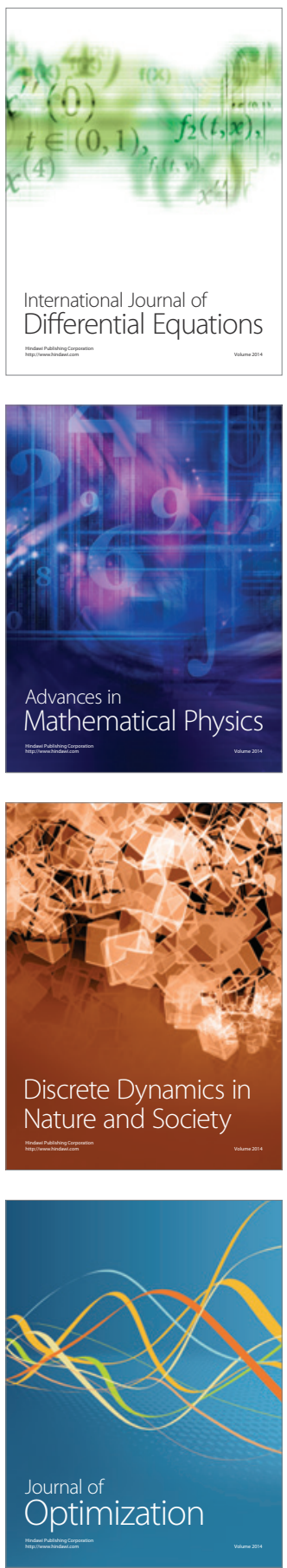